\title{
Is a further reduction of radiation exposure in scoliosis monitoring achievable using flat detector and pulsed fluoroscopy technology? - A feasibility study on human specimen
}

\section{Christian Walter ( $\nabla$ Christian.Walter@med.uni-tuebingen.de )}

Universitatsklinikum Tubingen Universitatsklinik fur Orthopadie https://orcid.org/0000-0003-37246533

\section{Juergen F. Schaefer}

University of Tuebingen, Department of Diagnostic and Interventional Radiology

Ilias Tsiflikas

University of Tuebingen, Department of Diagnostic and Interventional Radiology

\section{Research article}

Keywords: scoliosis, digital imaging, radiation dose reduction, fluoroscopy

Posted Date: July 21 st, 2020

DOI: https://doi.org/10.21203/rs.3.rs-41948/v1

License: (c) (i) This work is licensed under a Creative Commons Attribution 4.0 International License.

Read Full License 


\section{Abstract}

Background: In the case of scoliosis therapy, frequent radiological monitoring of the spine is necessary. However, x-ray requires high radiation doses; therefore digital pulsed fluoroscopy with flat detector technique can be used alternatively. The latest developments in this technology lead to further dose reduction with an improved image quality. To evaluate the new system, we asked if there is a difference in dose area product (DAP) concerning the opening area (OA) and image quality settings (IQS). Further we wanted to investigate the inter-observer reliability using an established scoring system and correlate the DAP with the point value.

Methods Therefore, we examined 4 cadaver spines ( $\mathrm{T} 1$ to sacrum) with the flat detector technique using digital pulsed fluoroscopy and simulated the abdominal soft tissues with water bags. The images were merged calculating whole spine images from several digital single images and evaluated by 3 different investigators (spine surgeon, experienced pediatric radiologist, assistant physician) using an established scoring system. For comparison and validation of our model, we used digital radiography images of the cadaver spines.

Results The values for the DAP increased from the small OA $\left(33 \% ; 0.56 \mu \mathrm{Gy} \cdot \mathrm{m}^{2}\right)$ to the maximum OA $\left(100 \% ; 0.82 \mu \mathrm{Gy} \cdot \mathrm{m}^{2}\right)$ by $45 \%(\mathrm{p}=.003)$ and from low IQS $\left(0.57 \mu \mathrm{Gy} \cdot \mathrm{m}^{2}\right)$ to high IQS $\left(0.84 \mu \mathrm{Gy} \cdot \mathrm{m}^{2}\right)$ by $48 \%$ $(p=.028)$. The inter-observer reliability was strong (3 vs. $1: \rho=.818 ; 3$ vs. $2: p=.742 ; 2$ vs. $1: \rho=.586 ; p$ $<.001)$, but there was no correlation between DAP and point value $(\rho=-.053, p=.588)$. Despite the low DAP, the setting $33 \%$ OA achieved the best point values, therefore this setting is preferred.

Conclusions Using a digital fluoroscopy system allows a significant reduction of radiation exposure for whole spine images by a factor of $7.5\left(3.88 \mu \mathrm{Gy} \cdot \mathrm{m}^{2}\right.$ to $\left.0.5 \mu \mathrm{Gy} \cdot \mathrm{m}^{2}\right)$ compared to slot-scanning $x$-ray (EOS).

\section{Introduction}

Adolescent idiopathic scoliosis (AIS) is a common spinal deformity, defined as a curvature of more than $10^{\circ}$ of the spine in the coronal plane accompanied by a rotation component (1). Conservative therapy with bracing and physiotherapy are common treatment options all over the world (2).

AIS usually requires annual full spine radiography during conservative treatment, because the deformity can progress until skeletal maturity is reached (3). This monitoring leads to elevated radiation dose during childhood and especially during adolescence, when the bones are growing at an accelerated rate. In earlier days, before the introduction of digital x-ray devices, cumulative doses of $140 \mathrm{mGy}$ could be observed on the female breast. This radiation exposure even led to an increased breast cancer rate in scoliosis patients $(4,5)$. Thanks to a number of technical developments such as digital x-ray using multiwire proportional chambers (6), radiation exposure has been reduced in recent decades by the ratio 13.1 for spinal examination (7). 
Digital x-ray diagnostics represent the standard of investigation to date, but this requires quit high radiation doses. In a previous study, using a conventional screen/film system with amplification factor

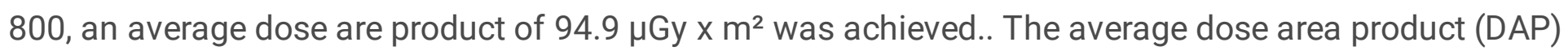
of the conventional, digital film-foil combination was $94.9 \mu \mathrm{Gy} \times \mathrm{m}^{2}(8)$.

Alternatively, using the flat detector technique with the whole spine image being calculated from several digital single images, a DAP of $9.37 \mu \mathrm{Gy} \times \mathrm{m}^{2}$ was achieved (9). A variant of the described technique is the storage of the pulsed fluoroscopy during the scanning process, yielding a DAP of $7.8 \mu \mathrm{Gy} \times \mathrm{m}^{2}(8)$.

The Multi Diagnost Eleva system (Philips Medical Systems; Netherlands) with a new flat detector, gridcontrolled fluoroscopy (GCF) and Dose Wise technology (10) was originally developed for pediatric applications such as cardiac and vascular interventions. Adapted to musculoskeletal examinations, this system provides the technical opportunity to further reduce the radiation exposure for the mostly young scoliosis patients.

Aim of the study was to evaluate the flat detector technique for whole spine examinations with pulsed fluoroscopy and determine the examination parameters for clinical use. Therefore, we investigated 4 human spinal specimens and evaluated the images by three investigators. First, we asked if differences in DAP depending on the opening area and image quality setting are assessable. Further we wanted to investigate the inter-observer reliability using an established scoring system and correlate the DAP with the point value. Finally, we wanted to establish the optimal settings for further human studies.

\section{Materials And Methods}

\section{Specimens}

We used four human spinal specimens for the tests, anonymously donated by body donors after a corresponding declaration of consent. The ethics vote with the number 891/2019B2 of the Ethics Committee of Tuebingen was available. In order to simulate the abdominal soft parts, a water bag of approximately seven liters was fixed on the specimens.

\section{Digital fluoroscopy:}

All examinations were carried out on a C-arc-equipped flat detector unit (Philips MultiDiagnost Eleva, Philips Medical Systems; Netherlands) with technical optimization of the unit for paediatric fluoroscopy purposes, including filtration and automatic customization of the X-ray beam spectrum, shape, and pulse frequency (10). The specimens described above were placed on the examination table in a plastic box 
including a reference ruler in the back of the specimen and examined by an experienced pediatric radiologist. During a synchronized scan with a C-arm speed of $4 \mathrm{~cm} / \mathrm{sec}$ fluoroscopic images were achieved with a pulsed frequency of 7.5 images per second. The images were merged by the software ViewForum R6.3 "Spine Measurements" (Philips Medical Systems, Netherlands) and an overall image was calculated based on the reference ruler (see Fig. 1A.).

See figure_1.jpg.

Figure 1: Images of specimen No. A 96. A Fluoroscopic image with opening area 33\%, image quality high B Digital x-ray image.

In preliminary tests, the manufacturer's recommendations of 7.5 images per second and minimum $50 \%$ opening area were tried to reduce. However, with a reduced pulsation of only 4 images per second or an opening area of $25 \%$, the image quality was no longer sufficient for the automatic image calculation.

Therefore we defined the following parameters for testing within the study: Opening area $100 \%, 50 \%$ and $33 \%$ and image quality setting - (low), o (medium), + (high). Each OA setting was combined with every image quality setting, resulting in 9 settings per specimen. All other parameters have been adopted identically from previous studies $(8,9)$ : Frame rate 7.5 images/sec; tube voltage $60 \mathrm{kV}$, tube current 250 $\mathrm{mA}$, filters: $1 \mathrm{~mm}$ Aluminum (Al), $0.1 \mathrm{~mm}$ copper (Cu), $0.3 \mathrm{~mm}$ copper. During the investigation, the dose area product was determined using a dose area product meter (Diamentor CD, PTW Dosimetry, Freiburg, Germany).

\section{Digital X-ray:}

For comparison, standardized whole spine radiographs of the spinal specimens were taken (see Fig 1 B), with a digital X-ray unit Digital Diagnost (Philips Medical Systems; Netherlands) on a dedicated grid stand for whole spine recordings, using a digital x-ray detector Pixium 4600 (Trixell, Moirans, France) with 
the standard examination protocol used in daily routine (tube voltage $85 \mathrm{kVp}$, automatic tube current modulation and an Exposure Index of 800).

\section{Image evaluation:}

The evaluation was carried out by three independent investigators (spine surgeon, experienced pediatric

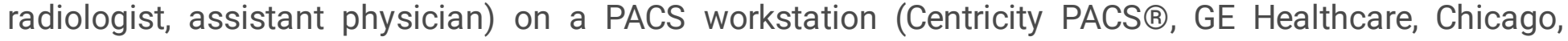
Illinois, United States), who were blinded to the settings of the fluoroscopic system. An established scoring system was utilized to evaluate the images, as described by Kloth et. al (11). In this system four evaluation criteria (endplates, pedicle, spinous process and lateral alignment) are each rated with a score from 1 (fully assessable) to 4 (not assessable) (see Tab. 1). All common tools for image diagnosis were available.

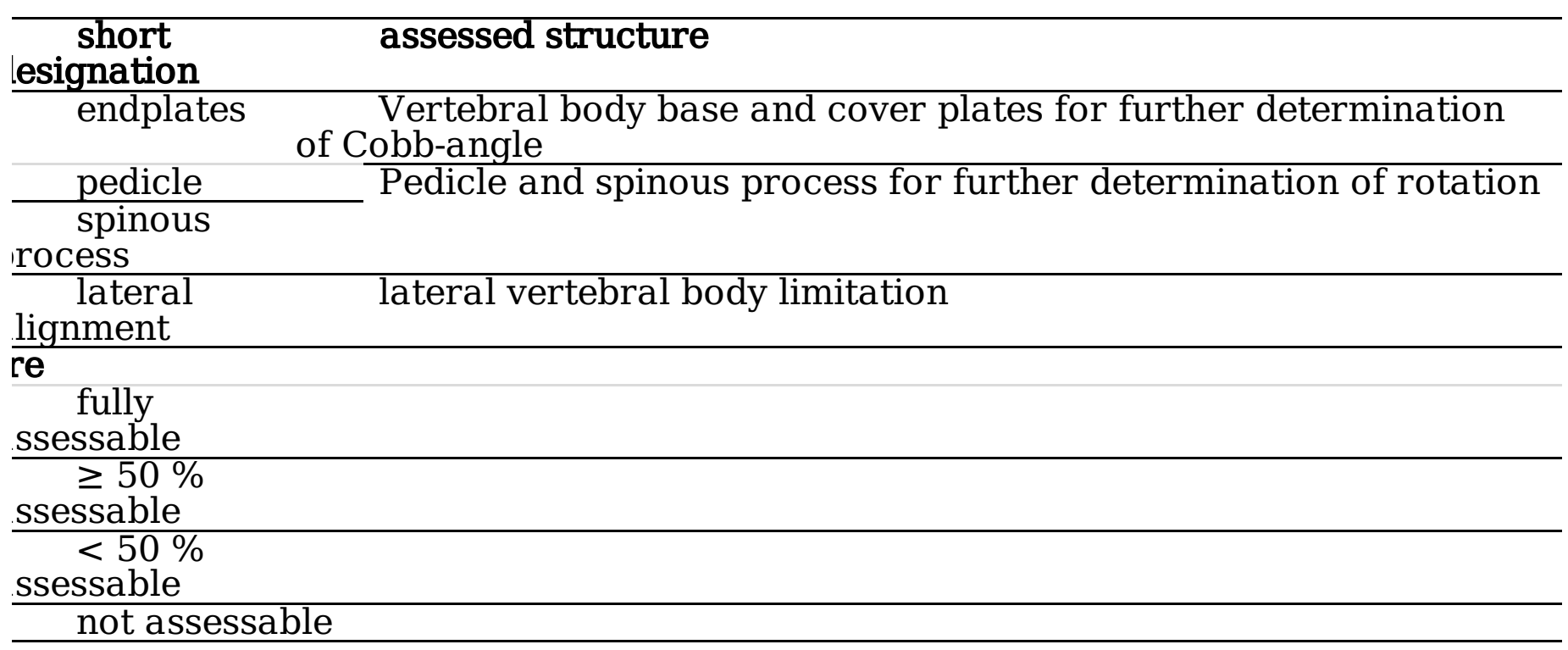

Tab. 1: Image Assessment Score described by Kloth et. al (11).

\section{Model validation:}


An important factor before the evaluation of the data was the validation of our model with cadaver specimen using digital x-ray: On average we found a DAP of $30.3 \mu \mathrm{Gy} \cdot \mathrm{m} \square$ for digital X-ray imaging in our study, corresponding to the data in patients' $x$-ray described in the literature (Kluba et al.: 31.5 pGy·ml) (12).

\section{Data analysis}

For data analysis, a sum score was calculated from the determined score points, leading to a minimum score value of 4 points (optimal assessment of the image) and a maximum score value of 16 points (image not assessable). This sum score was used for the further statistical calculation utilizing SPSSStatistics (IBM, Version 25.0.0.1). Comparing image quality settings paired t-tests were used and the pvalues were adjusted in accordance with the Bonferroni correction for multiple testing. Spearman correlation was used for inter-observer reliability. A significance level of $p=0.05$ was chosen for all tests and descriptive statistics were applied.

\section{Results}

In total, we scanned 9 different settings (OA: $33 \%, 50 \%$ and 100\%, image quality: $+, 0,-)$ for each spine specimen $(\mathrm{N}=4)$ resulting in 36 examinations with digital fluoroscopy. The automatic image generation worked in all 36 examinations. On average, 24.6 images (standard deviation +/- 5.6) were generated per examination. The mean value of the DAP over all examinations was $0.70 \mu \mathrm{Gy} \cdot \mathrm{m}^{2}$ with a standard deviation of $\pm 0.30 \mu \mathrm{Gy} \cdot \mathrm{m}^{2}$.

For the comparison images with digital X-ray we found a mean value of DAP of $30.32 \mu \mathrm{Gy} \cdot \mathrm{m}^{2}$ with a standard deviation of $\pm 1.1 \mu \mathrm{Gy} \cdot \mathrm{m}^{2}$.

First we asked if there is a difference in DAP depending on the opening area and image quality setting. We therefore analyzed the 36 measurements of the DAP and grouped them after the opening area setting $(100 \%, 50 \%$ and $33 \%)$. There were no outliers in the data and the Shapiro-Wilk test showed a normal distribution of the measured values ( $p>0.05$ in all 3 groups) (see figure $2 A$ ). The values for the DAP increased from the small opening area $\left(33 \%\right.$, Mean $(M)=0.56 \mu \mathrm{Gy} \cdot \mathrm{m}^{2}$, Standard-Deviation $\left.(\mathrm{SD})=.17\right)$ 
over $50 \%$ opening area $\left(M=0.70 \mu \mathrm{Gy} \cdot \mathrm{m}^{2}, \mathrm{SD}=.22\right)$ to the maximum opening area $(100 \% ; M=0.82$ $\left.\mu G y \cdot m^{2}, S D=.25\right)$ by $45 \%$ (see Table $\left.2 a\right)$.

\section{a}

\begin{tabular}{|c|c|c|c|c|c|c|}
\hline & $\mathrm{N}$ & Mean DAP & $\begin{array}{l}\text { Standard } \\
\text { deviation }\end{array}$ & Minimum & Maximum & Median \\
\hline $100 \%$ & 12 & 0.82 & 0.26 & 0.46 & 1.36 & 0.89 \\
\hline $50 \%$ & 12 & 0.70 & 0.22 & 0.39 & 1.02 & 0.72 \\
\hline $33 \%$ & 12 & 0.56 & 0.17 & 0.30 & 0.81 & 0.59 \\
\hline
\end{tabular}

b

\begin{tabular}{|c|c|c|c|c|c|c|}
\hline & $\mathrm{N}$ & Mean DAP & $\begin{array}{l}\text { Standard } \\
\text { deviation }\end{array}$ & Minimum & Maximum & Median \\
\hline- & 12 & 0.57 & 0.22 & 0.30 & 1.00 & 0.48 \\
\hline o & 12 & 0.67 & 0.15 & 0.47 & 1.00 & 0.63 \\
\hline+ & 12 & 0.84 & 0.26 & 0.39 & 1.36 & 0.86 \\
\hline
\end{tabular}

Table 2: DAP values in $\mu \mathrm{Gy} \cdot \mathrm{m}^{2}$ for the settings (a) opening area and (b) image quality.

In addition, the individual pairs were compared using a paired t-test and the p-values were adjusted in accordance with the Bonferroni correction for multiple testing. Finally we calculated the effect strengths (Cohens $d_{z}$ ) with the formula $d_{z}=\left(M_{1}-M_{2}\right) / S D$. $|d|=0,2$ means small effect, $|d|=0,5$ means medium effect, $|d|=0,8$ means strong effect. The first pair $50 \%$ vs. $33 \%$ OA showed significant differences with strong effect $\left(t(11)=9.75, p<.001, d_{z}=2.81\right)$, same in second pair $100 \%$ vs. $33 \%(t(11)=4.82, p=0.003$, $\left.d_{z}=1.30\right)$. No significant change after Bonferroni correction with medium effect size was found in $100 \%$ vs. $50 \% \mathrm{OA}\left(t(11)=2.28, p=0.132, \mathrm{~d}_{\mathrm{z}}=1.30\right)$ (see figure $\left.2 \mathrm{a}\right)$.

See figure_2.jpg

Figure 2: Dose area product in $\mu \mathrm{Gy} \cdot \mathbf{m} l$. Boxplots displaying the Dose area product $[\mu \mathrm{Gy} \cdot \mathrm{m} \square]$ measured in the different settings of fluoroscopy. (a) Opening area in\%, (b) Image 
quality setting. The data show a clear trend: A large opening area and a + setting of the image quality. $*$ denotes significant differences $(\mathrm{p}<0.05)$ after paired $\mathrm{t}$-test and Bonferroni correction for multiple testing.

Subsequently, we performed the same procedure for image quality setting and found an increase of DAP from the low image quality setting $\left(M=0.57 \mu \mathrm{Gy} \cdot \mathrm{m}^{2}, S D=0.22\right)$ over the medium setting $(M=0.67$ $\left.\mu G y \cdot m^{2}, S D=0.15\right)$ to the high setting $\left(M=0.84 \mu \mathrm{Gy} \cdot \mathrm{m}^{2}, S D=0.26\right)$ by $48 \%$.

The first pair o vs. + showed significant differences with strong effect $\left(t(11)=-2.86, p=0.045, d_{z}=0.85\right)$. The other pairs showed no significant differences: - vs. $+\left(t(11)=-2.53, p=0.084, d_{z}=0.73\right)$ and o vs. $\left(t(11)=-1.53, p=0.465, d_{z}=0.45\right)$ (see figure $\left.2 b\right)$.

In conclusion, we can state that there is an increase in DAP from small to large aperture area and from low to high image quality, even if not all differences have become significant.

Finally, we wanted to establish the optimal settings for further human studies. We therefore analyzed the 108 point scores resulting from the scoring of 36 examinations through 3 observers and grouped them by opening area setting $(100 \%, 50 \%$ and $33 \%)$. The point score improved from $100 \%$ OA (Median (MED) $=$ 9.5) over $50 \%$ OA (MED = 8) to $33 \%$ OA (MED = 7). The data showed no normal distribution in the Shapiro-Wilk test). Due to the lack of a normal distribution, we used the non-parametric Friedman's test. The three settings $(100 \%, 50 \%$ and $33 \%)$ showed significant changes in point score $\left(\right.$ Friedman test $\chi^{2}(2)=$ $40.32, p<0.001, n=36$ ). Subsequently the results of Friedman's test underwent Dunn's pairwise post hoc tests and Bonferroni adjustments of the p-values. Significant differences were found between all settings (100vs.50 p=0.01, 50vs.33 $p=0.001,100 v s .33 p<0.001)$.

The effect sizes $(r)$ were calculated using the formula $r=z / \sqrt{ } n(z=$ test statistic, $n=$ number of pairs). A strong effect $(r>0.50)$ was found in $100 \%$ vs. $33 \% \mathrm{OA}(r=.8)$ and a medium effect $(0.3 \leq r<0.5)$ in $100 \%$ vs. $50 \% \mathrm{OA}(r=0.40)$ and $50 \%$ vs. $33 \%(r=.4)$. Next we grouped the data by image quality setting $(-, 0,+)$ and repeated the procedure. The point score improved from low image quality setting (MED = 9) over medium setting $(M E D=8)$ to high setting $(M E D=7)$. The Friedman test showed again significant 
changes in point score $\left(X^{2}(2)=22.51, p<0.001, n=36\right.$ ). Significant differences in the post hoc analyze were found between + and - setting $(p<0.001 ; r=0.43)$, as well as + and $o(p=0.004 ; r=0.55)$. Looking at the results of $O A$ and image quality in combination, the setting $33 /+$ shows the best point scores in the image assessment (see figure 5).

See figure_5.jpg

Figure 5: Scatter plot with jittering of the different settings depending on the determined point values (Kloth score). The maximum value was 4 points (optimal assessment of the image), the minimum value 16 points (evaluation not possible).

\section{Discussion}

Our study shows a very interesting result: A low OA of $33 \%$ leads to a reduction in radiation exposure compared with a large OA (100\%) of $45 \%$ and at the same time to an improved assessment of the image, meaning 33\% OA setting can simultaneously improve the image assessment and reduce the radiation dose. Looking at the image quality setting, we found the expected correlation of improved image assessment from - to + with increasing radiation exposure.

Three radiological applications are currently available for monitoring the course of scoliosis patients: First, the fluoroscopic system presented here, which is rarely used in clinical routine. Second, digital x-ray examinations, representing the standard examination tool in most clinics. Third, the slot-scanning x-ray (EOS), which is increasingly used due to the three-dimensional images. In the following, these examination systems will be compared with the Philips MultiDiagnost Eleva and our results.

Comparing our fluoroscopic system with digital x-ray diagnostics, fluoroscopy shows a significantly reduced radiation exposure. In recent years the development and spread of digital image processing systems and flat detectors, which have higher detectable quantum efficiency (DQE) led to a lower mean DAP compared to conventional film-foil systems. Nevertheless, in previous studies, fluoroscopy was clearly superior concerning radiation exposure compared to x-ray diagnostics. In a previous study in our departments, we could show significantly reduced DAP values for fluoroscopy $\left(7.8 \mu \mathrm{Gy} \cdot \mathrm{m}^{2}\right)$ compared to $x$-ray imaging $\left(94.9 \mu \mathrm{Gy} \cdot \mathrm{m}^{2}\right)(8)$. However, other working groups were able to reduce the DAP of anteriorposterior x-ray images using a digital flat panel detector (FPD) and an image stitching system (ISS) in full-spine radiography to $16.8 \mu \mathrm{Gy} \cdot \mathrm{m}^{2}$ (13). Comparing conventional films (DAP: $97.0 \mu \mathrm{Gy} \cdot \mathrm{m}^{2}$ ), digital xray $\left(31.5 \mu \mathrm{Gy} \cdot \mathrm{m}^{2}\right)$ and digital fluoroscopy $\left(5.0 \mu \mathrm{Gy} \cdot \mathrm{m}^{2}\right)$, Kluba et al. found similar results (12). Compared to this information from the literature, our examination technique reveals enormous possibilities of 
reducing the radiation exposure by a factor of $33\left(16.8 \mu \mathrm{Gy} \cdot \mathrm{m}^{2}\right.$ to $\left.0.5 \mu \mathrm{Gy} \cdot \mathrm{m}^{2}\right)$ compared to the latest $\mathrm{x}$ ray diagnostics.

First advantage for x-ray examinations is the standardized feasibility by the radiology assistant according to generally accepted guidelines and settings (14). To date, there is no automated protocol for the scanning process with the fluoroscopic system and so far has to been carried out by the radiologist.

The second advantage of the x-ray examinations is a better assessability with higher score points compared to the fluoroscopic technique. However, the end plates as well as the spinous processes are particularly relevant for the assessment of a deformity on the spine. These are already sufficiently displayed at a fluoroscopic image and do not require the high assessability and radiation dose of x-ray such as traumatic or oncological issues. From the authors' point of view, an x-ray examination would be helpful at the initial presentation to rule out malformations or dysplasia. Further monitoring of the deformity could be carried out with the fluoroscopic system.

Another method that has become increasingly popular in recent years is slot-scanning x-ray (EOS), which is used for examinations in scoliosis patients (15). First advantage of EOS is a software called sterEOS 3D, which offers the opportunity of visualizing the spinal deformity in all three planes (16). Second advantage is that it is even easier to use than X-rays. However, compared with our results, high radiation exposure was described in DAP $\left(59.9 \mu \mathrm{Gy} \cdot \mathrm{m}^{2}\right.$ ap in females and $18.2 \mu \mathrm{Gy} \cdot \mathrm{m}^{2}$ in children in an anteriorposterior view, regarding a Phantom Study (17). In accordance with the ALARA principle (as low as reasonably achievable), a reduction in radiation exposure was already sought for the EOS system and DAP of $3.88 \mu \mathrm{Gy} \cdot \mathrm{m}^{2}$ in female $4.42 \mu \mathrm{Gy} \cdot \mathrm{m}^{2}$ in male was achieved (18).

Despite the significant improvements and further reduction of DAP in EOS technology, our system can reduce DAP again by a factor of $7.5\left(3.88 \mu \mathrm{Gy} \cdot \mathrm{m}^{2}\right.$ to $\left.0.5 \mu \mathrm{Gy} \cdot \mathrm{m}^{2}\right)$.

\section{Study Limitations}

A limitation of our study is certainly the use of cadaver and an evaluation with patients is still pending. However, we were able to show that our model achieved absolutely similar values compared to data from routine clinical practice (x-ray) and from literature (Our results: $30.3 \mu \mathrm{Gy} \cdot \mathrm{m}^{2}$ for digital X-ray imaging vs. Kluba et al.: $31.5 \mu \mathrm{Gy} \cdot \mathrm{m}^{2}$ ) [12]. Nevertheless, the age and height of the patient are relevant influencing factors on the DAP, which cannot be fully mapped with the specimen of the body donor.

\section{Conclusion}

From the authors' point of view, the fluoroscopic system presented in this paper shows excellent radiation exposure values with good image assessability in the setting 33\% OA / + image quality, leading to great benefit especially for young scoliosis patients. However, the practical feasibility must be improved e.g. by automated scanning processes, comparable with the EOS system. 


\section{Declarations}

Ethics approval and consent to participate

The preparations were administered by University of Tuebingen, Department for Anatomy (ElfriedeAulhorn-Straße, 72076 Tuebingen, Germany) with documented consent obtained from the individual who has donated their body for research or scientific purposes. The positive vote of the ethics committee (ethic committee medical faculty of the University of Tuebingen) with the number 891/2019BO2 is available, includes ethical approval for the use of cadavers within the study.

Consent for publication

Not applicable.

Availability of data and materials

The datasets used and/or analyzed during the current study are available from the corresponding author on reasonable request.

Competing interests

The authors declare that they have no competing interests.

Funding

The study was funded by the department's internal resources without external sponsorship.

Authors' contributions:

$\mathrm{CW}$ was one of the observers and wrote the manuscript. JS developed the study design and co-wrote the manuscript. IT was one of the observers and performed the measurements. All authors read and approved the final manuscript.

Acknowledgements

We acknowledge support by Deutsche Forschungsgemeinschaft and Open Access Publishing Fund of University of Tübingen.

\section{References}

1. Weinstein SL, Dolan LA, Cheng JC, Danielsson A, Morcuende JA. Adolescent idiopathic scoliosis. Lancet. 2008;371(9623):1527-37.

2. Weinstein SL, Dolan LA, Wright JG, Dobbs MB. Effects of bracing in adolescents with idiopathic scoliosis. N Engl J Med. 2013;369(16):1512-21. 
3. Minehiro K, Demura S, Ichikawa K, Sasagawa T, Takahashi N, Minami S, et al. Dose Reduction Protocol for Full Spine X-ray Examination Using Copper Filters in Patients With Adolescent Idiopathic Scoliosis. Spine (Phila Pa 1976). 2019;44(3):203-10.

4. Hoffman DA, Lonstein JE, Morin MM, Visscher W, Harris BS 3rd, Boice JD. Jr. Breast cancer in women with scoliosis exposed to multiple diagnostic x rays. J Natl Cancer Inst. 1989;81(17):1307-12.

5. Doody MM, Lonstein JE, Stovall M, Hacker DG, Luckyanov N, Land CE. Breast cancer mortality after diagnostic radiography: findings from the U.S. Scoliosis Cohort Study. Spine (Phila Pa 1976). 2000;25(16):2052-63.

6. Charpak G. Electronic Imaging of Ionizing-Radiation with Limited Avalanches in Gases. Usp Fiz Nauk+. 1993;163(10):57-66.

7. Kalifa G, Charpak Y, Maccia C, Fery-Lemonnier E, Bloch J, Boussard JM, et al. Evaluation of a new low-dose digital $x$-ray device: first dosimetric and clinical results in children. Pediatr Radiol. 1998;28(7):557-61.

8. Schaefer J, Kluba T, Niemeyer T, Hahnfeldt T, Vonthein R, Kottke R, et al. [Comparison of conventional full spine radiographs and fluoroscopic scanning method in young patients with idiopathic scoliosis]. Rofo. 2005;177(8):1110-5.

9. Geijer H, Beckman K, Jonsson B, Andersson T, Persliden J. Digital radiography of scoliosis with a scanning method: initial evaluation. Radiology. 2001;218(2):402-10.

10. Stueve D. Management of pediatric radiation dose using Philips fluoroscopy systems DoseWise: perfect image, perfect sense. Pediatr Radiol. 2006;36(Suppl 2):216-20.

11. Kloth JK, Wiedenhoefer B, Stiller W, Burkholder I, Kauczor HU, Ewerbeck V, et al. [Modern digital plainradiography of the whole spine in scoliosis patients-dose reduction and quality criteria]. Rofo. 2013;185(1):48-54.

12. Kluba T, Schafer J, Hahnfeldt T, Niemeyer T. Prospective randomized comparison of radiation exposure from full spine radiographs obtained in three different techniques. Eur Spine J. 2006;15(6):752-6.

13. Grieser T, Baldauf AQ, Ludwig K. Radiation dose reduction in scoliosis patients: low-dose full-spine radiography with digital flat panel detector and image stitching system. Rofo. 2011;183(7):645-9.

14. Morvan G, Mathieu P, Vuillemin V, Guerini H, Bossard P, Zeitoun F, et al. Standardized way for imaging of the sagittal spinal balance. Eur Spine J. 2011;20(Suppl 5):602-8.

15. Begon M, Scherrer SA, Coillard C, Rivard CH, Allard P. Three-dimensional vertebral wedging and pelvic asymmetries in the early stages of adolescent idiopathic scoliosis. Spine J. 2015;15(3):477-86.

16. Amzallag-Bellenger E, Uyttenhove F, Nectoux E, Moraux A, Bigot J, Herbaux B, et al. Idiopathic scoliosis in children and adolescents: assessment with a biplanar X-ray device. Insights Imaging. 2014;5(5):571-83.

17. Damet J, Fournier P, Monnin P, Sans-Merce M, Ceroni D, Zand T, et al. Occupational and patient exposure as well as image quality for full spine examinations with the EOS imaging system. Med Phys. 2014;41(6):063901. 
18. Hui SC, Pialasse JP, Wong JY, Lam TP, Ng BK, Cheng JC, et al. Radiation dose of digital radiography (DR) versus micro-dose $x$-ray (EOS) on patients with adolescent idiopathic scoliosis: 2016 SOSORTIRSSD "John Sevastic Award" Winner in Imaging Research. Scoliosis Spinal Disord. 2016;11:46.

\section{Figures}

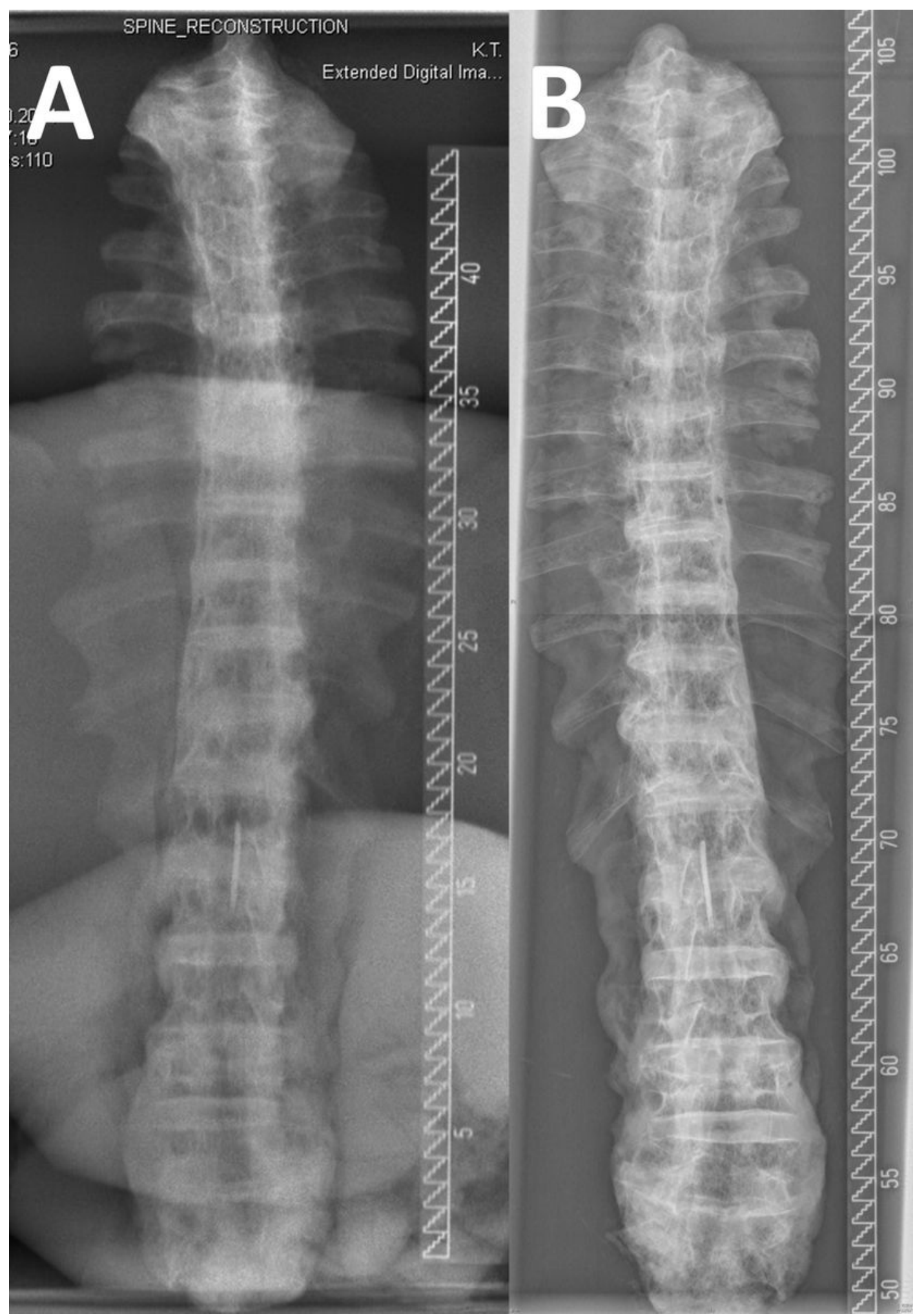

Figure 1 
Images of specimen No. A 96. A Fluoroscopic image with opening area 33\%, image quality high B Digital $x$-ray image.

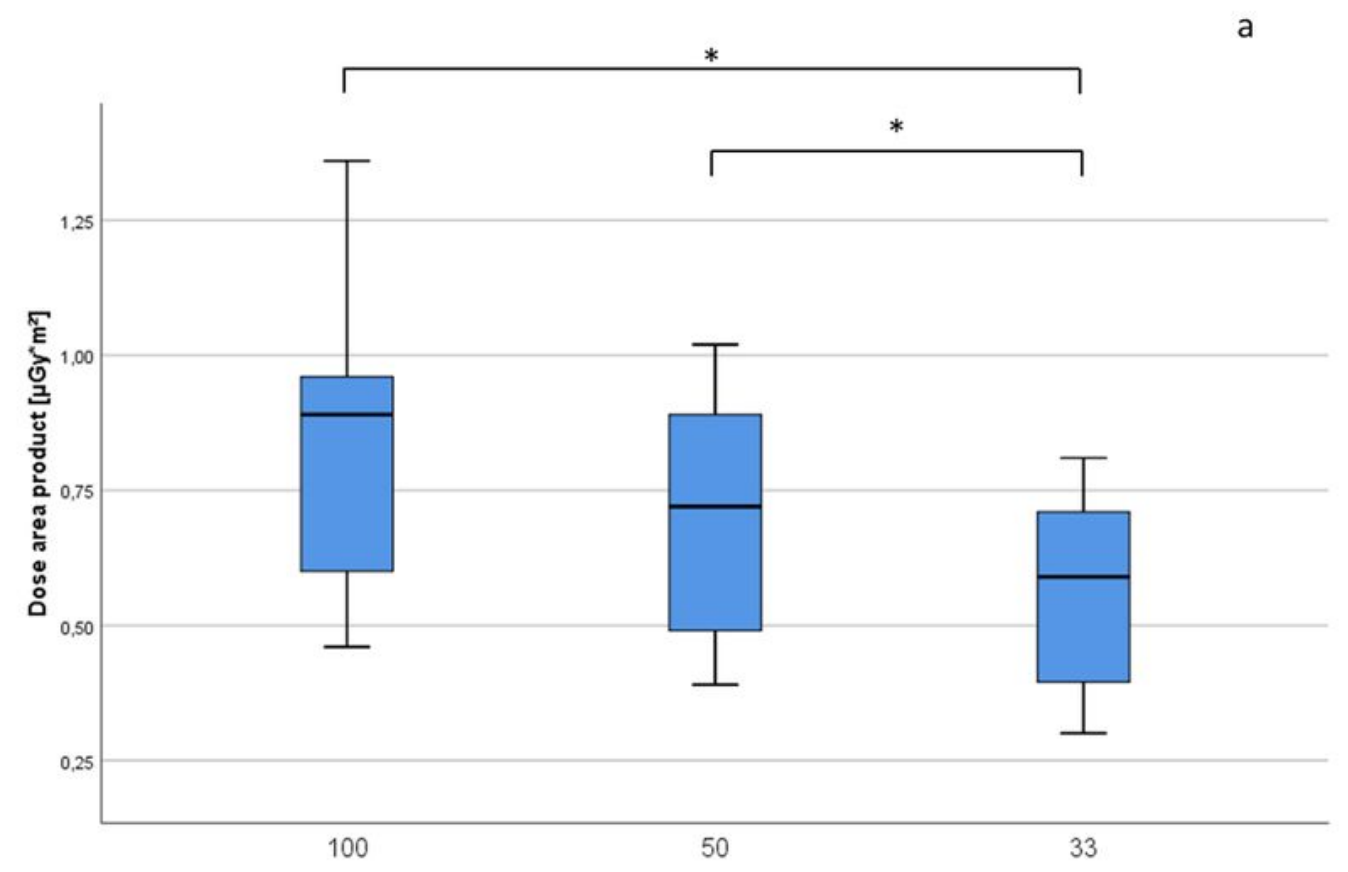

b

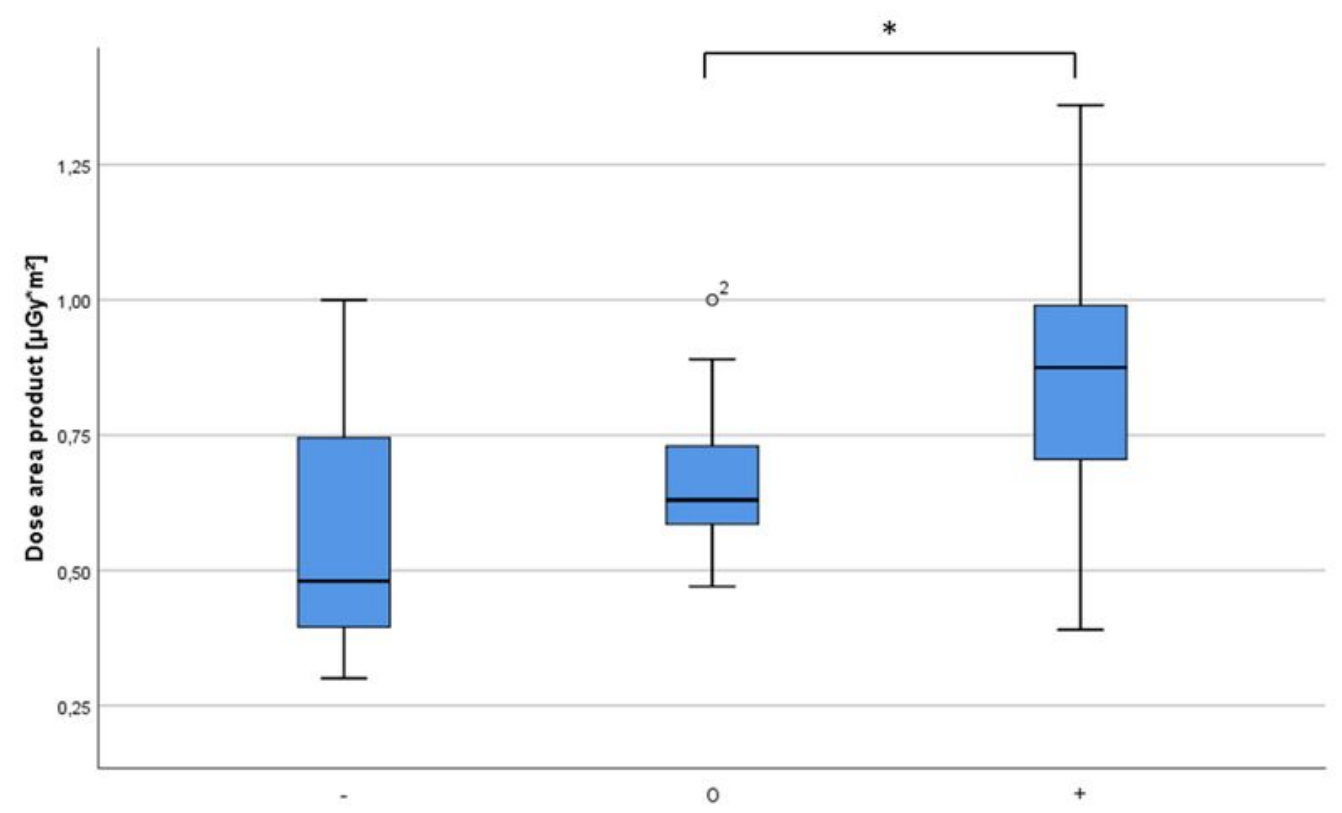

Figure 2

Dose area product in $\mu \mathrm{Gy} \cdot \mathrm{m}^{2}$. Boxplots displaying the Dose area product [ $\left.\mu \mathrm{Gy} \cdot \mathrm{m}^{2}\right]$ measured in the different settings of fluoroscopy. (a) Opening area in\%, (b) Image quality setting. The data show a clear 
trend: A large opening area and $a+$ setting of the image quality. * denotes significant differences $(p<$ $0.05)$ after paired t-test and Bonferroni correction for multiple testing.

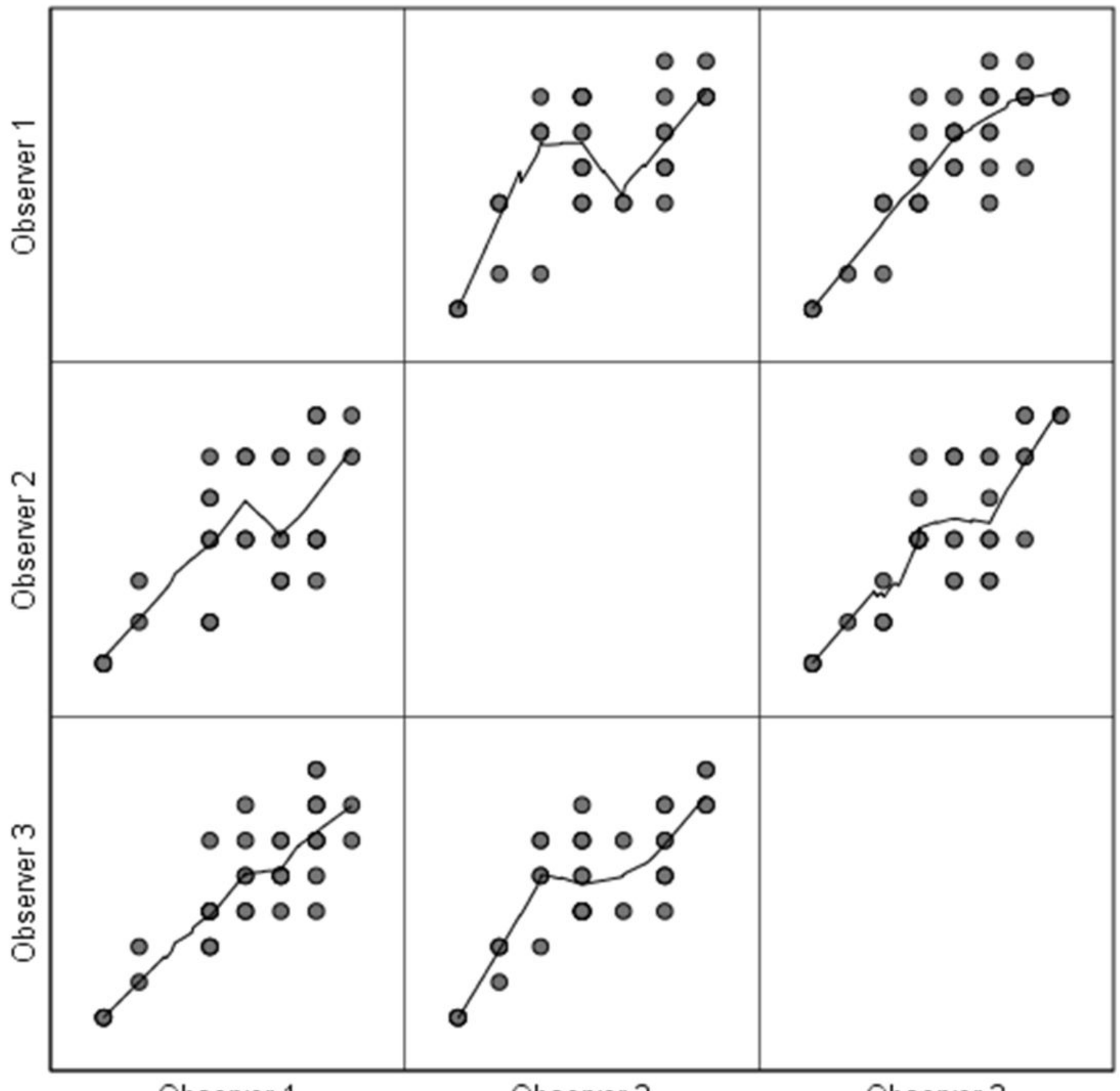

Observer 1

Observer 2

Observer 3

Figure 3

Scatterplots of different observers with LOESS smoothing. The inter-observer reliability was strong ( 3 vs. $1: \rho=.818 ; 3$ vs. $2: \rho=.742 ; 2$ vs. $1: \rho=.586 ; p<0.001)$. 


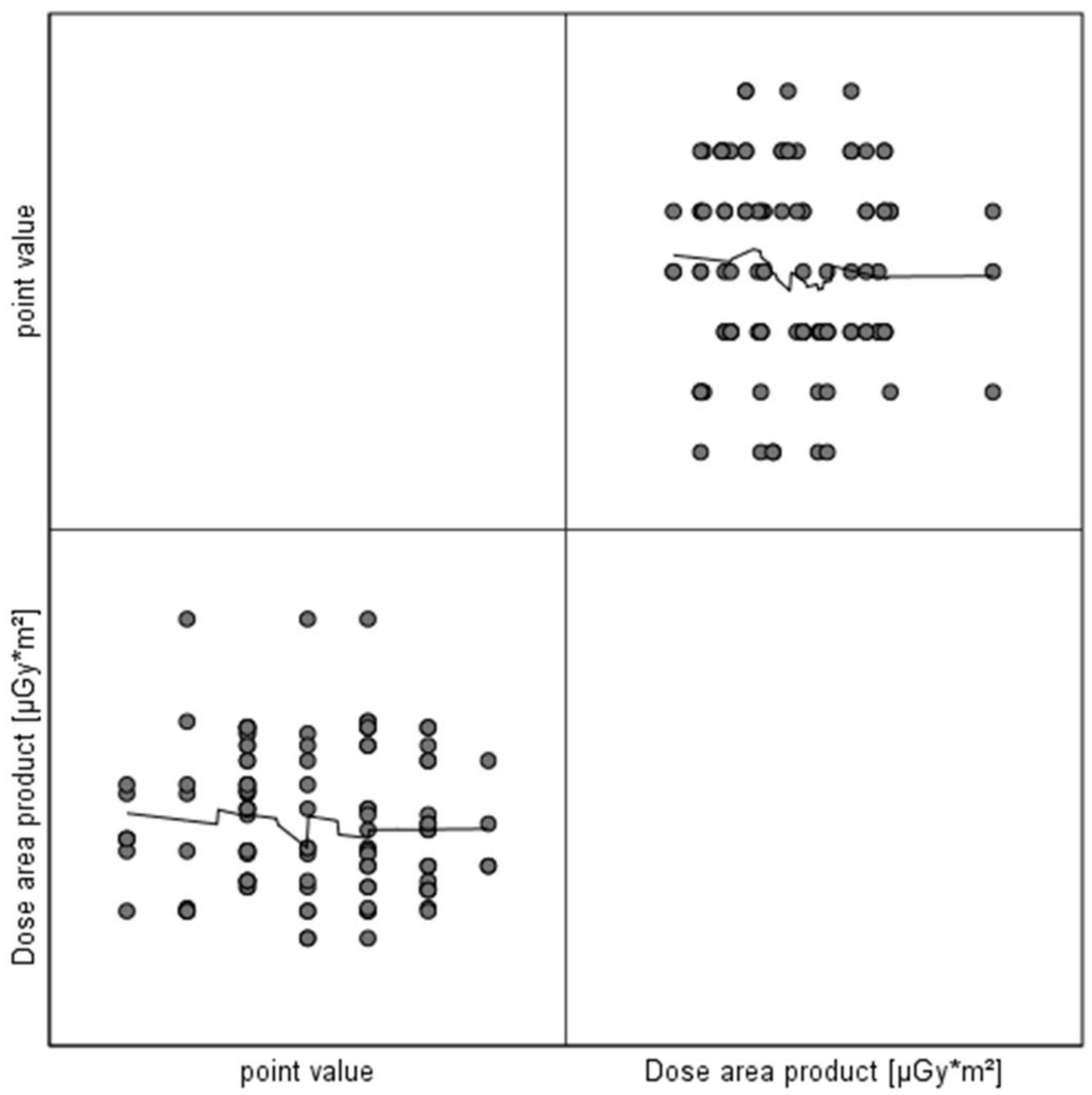

Figure 4

Scatterplots of Dose area product $\left[\mu \mathrm{Gy} \cdot \mathrm{m}^{2}\right]$ vs Kloth score value with LOESS smoothing. No correlation was found between DAP and point value. $(\rho=-.053, p=.588)$. 


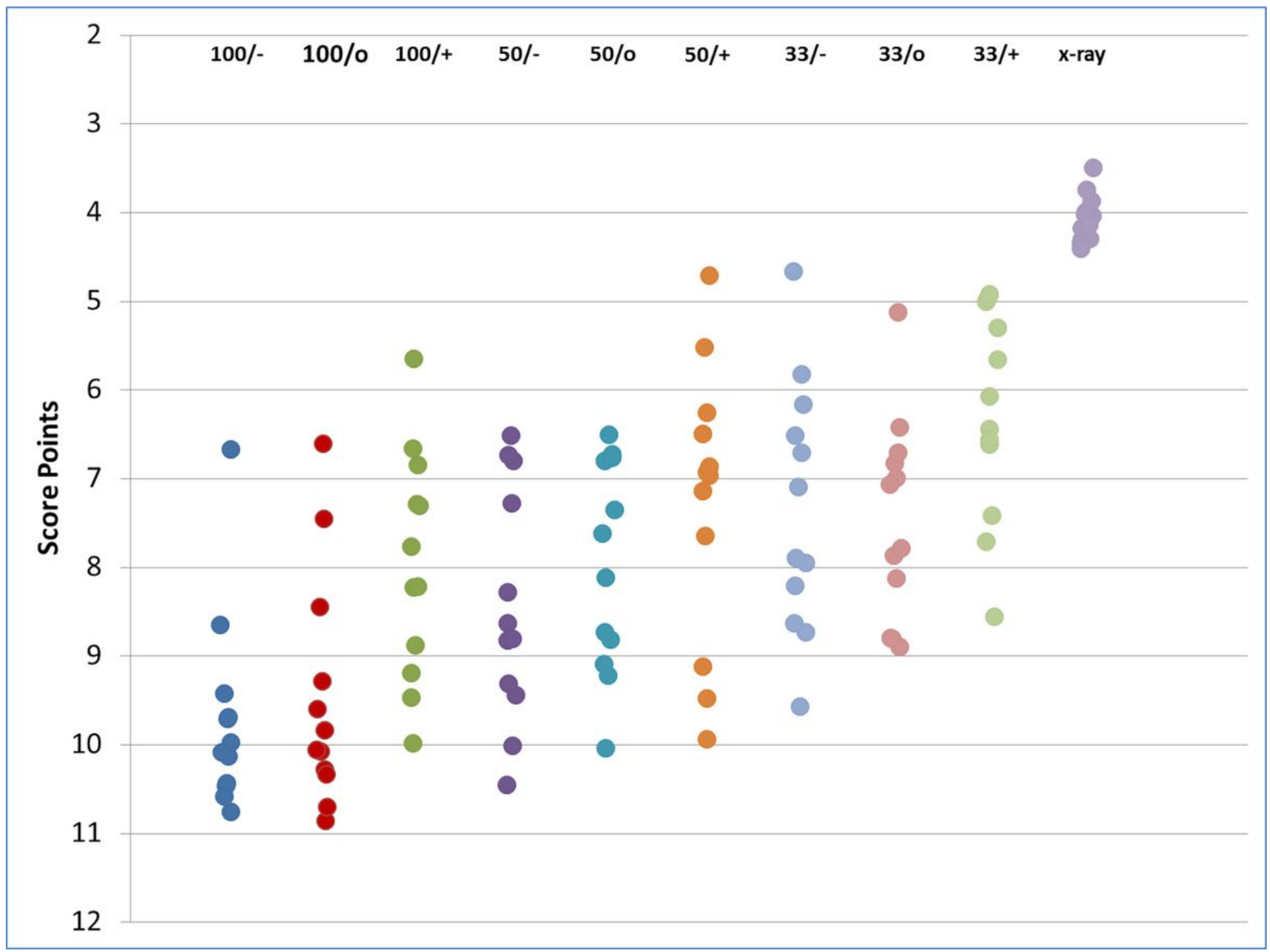

\section{Figure 5}

Scatter plot with jittering of the different settings depending on the determined point values (Kloth score). The maximum value was 4 points (optimal assessment of the image), the minimum value 16 points (evaluation not possible). 